\title{
Effects of a solar wind dynamic pressure increase in the magnetosphere and in the ionosphere
}

\author{
L. Juusola ${ }^{1,2}$, K. Andréeová ${ }^{3}$, O. Amm ${ }^{1}$, K. Kauristie ${ }^{1}$, S. E. Milan ${ }^{4}$, M. Palmroth ${ }^{1}$, and N. Partamies ${ }^{1}$ \\ ${ }^{1}$ Finnish Meteorological Institute, Helsinki, Finland \\ ${ }^{2}$ Department of Physics and Technology, University of Bergen, Bergen, Norway \\ ${ }^{3}$ Department of Physics, University of Helsinki, Helsinki, Finland \\ ${ }^{4}$ Department of Physics and Astronomy, University of Leicester, Leicester, UK
}

Received: 12 February 2010 - Revised: 20 August 2010 - Accepted: 20 October 2010 - Published: 27 October 2010

\begin{abstract}
On 17 July 2005, an earthward bound north-south oriented magnetic cloud and its sheath were observed by the ACE, SoHO, and Wind solar wind monitors. A steplike increase of the solar wind dynamic pressure during northward interplanetary magnetic field conditions was related to the leading edge of the sheath. A timing analysis between the three spacecraft revealed that this front was not aligned with the GSE y-axis, but had a normal $(-0.58,0.82,0)$. Hence, the first contact with the magnetosphere occurred on the dawnside rather than at the subsolar point. Fortunately, Cluster, Double Star 1, and Geotail happened to be distributed close to the magnetopause in this region, which made it possible to closely monitor the motion of the magnetopause. After the pressure front had impacted the magnetosphere, the magnetopause was perceived first to move inward and then immediately to correct the overshoot by slightly expanding again such that it ended up between the Cluster constellation with Double Star 1 inside the magnetosphere and Geotail in the magnetosheath. Coinciding with the inward and subsequent outward motion, the ground-based magnetic field at low latitudes was observed to first strengthen and then weaken. As the magnetopause position stabilised, so did the groundbased magnetic field intensity, settling at a level slightly higher than before the pressure increase. Altogether the magnetopause was moving for about $15 \mathrm{~min}$ after its first contact with the front. The high latitude ionospheric signature consisted of two parts: a shorter (few minutes) and less intense preliminary part comprised a decrease of $\mathrm{AL}$ and a negative variation of PC. A longer (about ten minutes) and more intense main part of the signature comprised an increase of $\mathrm{AU}$ and a positive variation of PC. Measurements from sev-
\end{abstract}

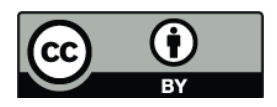

Correspondence to: L. Juusola (liisa.juusola@fmi.fi) eral ground-based magnetometer networks (210 MM CPMN, CANMOS, CARISMA, GIMA, IMAGE, MACCS, SuperMAG, THEMIS, TGO) were used to obtain information on the ionospheric $\boldsymbol{E} \times \boldsymbol{B}$ drift. Before the pressure increase, a configuration typical for the prevailing northward IMF conditions was observed at high latitudes. The preliminary signature coincided with a pair of reverse convection vortices, whereas during the main signature, mainly westward convection was observed at all local time sectors. Afterwards, the configuration preceding the pressure increase was recovered, but with slightly enhanced convection. Based on the timing analysis, the existence of the preliminary signature coincided with the passage of the oblique pressure front, whereas during the main signature the front was already well past Earth. The main signature existed during the time the magnetopause was observed to move. As the position stabilised, also the signature disappeared.

Keywords. Interplanetary physics (Interplanetary shocks) Magnetospheric physics (Magnetosphere-ionosphere interactions; Solar wind-magnetosphere interactions)

\section{Introduction}

The interplanetary counterpart of a coronal mass ejection (CME) consists of two distinct regions: the sheath and the ejecta. The sheath is defined as the region ahead of the ejecta, where proton temperature and density are enhanced, and the directional changes of the interplanetary magnetic field (IMF) are irregular. When the speed difference between the ejecta and the ambient solar wind exceeds that of the local magnetosonic speed, at which information is transferred in the plasma, a shock wave is formed upstream of the ejecta. In this case, the sheath refers to the region between the shock

Published by Copernicus Publications on behalf of the European Geosciences Union. 
and the ejecta. A subset of ejecta, called magnetic clouds, are identified by a smooth rotation of the large-scale magnetic field from northward to southward or from southward to northward, enhanced magnetic field magnitude, and decrease of plasma temperature (Burlaga et al., 1981; Klein and Burlaga, 1982). Both sheath and ejecta can be geoeffective (Pulkkinen et al., 2007). The Earth encounters sheaths more frequently than ejecta, as they extend farther.

On 17 July 2005, a magnetic cloud and its sheath were observed heading towards Earth. A steep increase of the solar wind dynamic pressure was observed at the leading edge of the sheath. In this study, we analyse the response of the magnetosphere-ionosphere system on the arrival of this pressure front. The fortunate positioning of several magnetospheric satellites allowed the close monitoring of the magnetopause position, while ground-based magnetic field and radar measurements yielded information on processes occurring inside the magnetosphere.

The main source of ground-based magnetic field disturbances at high latitudes are the ionospheric currents. Because the horizontal ionospheric currents are concentrated in a relatively thin layer between about $90-130 \mathrm{~km}$ altitude (e.g., Kamide and Brekke, 1977), they are often modelled as a spherical surface current density $\boldsymbol{J}(\theta, \phi)([J]=\mathrm{A} / \mathrm{m})$ at a constant altitude of about $100 \mathrm{~km}$. Here, $\theta$ and $\phi$ are the colatitude and longitude. Like any vector field, $\boldsymbol{J}$ can be divided into divergence-free (df) and curl-free (cf) components

$$
\begin{aligned}
\boldsymbol{J}(\theta, \phi) & =\mathbf{J}_{\mathrm{cf}}(\theta, \phi)+\boldsymbol{J}_{\mathrm{df}}(\theta, \phi) \\
\nabla \cdot \boldsymbol{J}_{\mathrm{df}} & =0 \\
\left(\nabla \times \boldsymbol{J}_{\mathrm{cf}}\right)_{\mathrm{r}} & =0,
\end{aligned}
$$

where the subscript " $\mathrm{r}$ " refers to the radial component. The field-aligned current density $\left(j_{\mathrm{r}}\right)$ is related to the divergence of the horizontal current density $j_{\mathrm{r}}(\theta, \phi)=-\nabla \cdot \boldsymbol{J}(\theta, \phi)$ $\left([j]=\mathrm{A} / \mathrm{m}^{2}\right)$, and hence, closed by its curl-free component. According to Fukushima (1976), the combined magnetic field of the curl-free component and (radial) field-aligned currents is confined to the region above the horizontal current layer. Thus, $\boldsymbol{J}_{\mathrm{df}}$ causes the same magnetic field below the ionosphere as the original 3-D distribution, consisting of $\boldsymbol{J}_{\mathrm{df}}$, $\boldsymbol{J}_{\mathrm{cf}}$, and $j_{\mathrm{r}}$, and is therefore also called the equivalent current density. For uniform conductances, the divergence-free and curl-free components would equal the Hall and Pedersen components, respectively. Assuming that there is no significant potential drop along the magnetic field lines, the Hall currents, which flow anti-parallel to the ionospheric $\boldsymbol{E} \times \boldsymbol{B}$ drift, can be mapped to the magnetospheric convection. The eastward and westward electrojets in the dusk and dawn sectors of the auroral oval, and the Harang discontinuity on the nightside, are the predominant features of this current component in the ionosphere. Thus, ground-based magnetic field measurements can give information on the magnetospheric convection.
The dynamic pressure regulates the size of the magnetosphere such that an increase of the pressure compresses the magnetopause. The compression is known to manifest itself as an enhancement of the ground-based magnetic field. Moreover, a sudden change of the solar wind dynamic pressure is believed to produce antisunward travelling convection vortices at high latitudes (e.g., Amm et al., 2002).

At least two theories have been proposed to explain the vortices: according to Kivelson and Southwood (1991), a solar wind pressure front establishes vortical flows on magnetopause flux tubes. The flows set up Alfvén waves that carry field-aligned current to the ionosphere, producing also in the ionosphere a pair of convection vortices of opposite polarisation. According to Glassmeier (1992), on the other hand, compression of the magnetopause leads to a modification of the magnetopause current, part of which is diverted to the ionosphere.

Iyemori and Araki (1982) found that the equivalent current configuration related to a sudden change in solar wind dynamic pressure during northward interplanetary magnetic field conditions is a single vortex encircling the cusp region. In the Northern Hemisphere, for a dynamic pressure increase, the equivalent current of the vortex was directed eastward. The related Auroral Electrojet index (AE, Davis and Sugiura, 1966) disturbance thus consisted of an increase in AU with small or no variation in AL.

Stauning and Troshichev (2008) examined the influence of the solar wind dynamic pressure on the Polar Cap index (PC, Troshichev et al., 1988) in cases of global sudden impulses or storm sudden commencements. The PC index is derived from polar magnetic variations (PCN index in the Northern Hemisphere and PCS index in the Southern Hemisphere) and is primarily a measure of the intensity of the transpolar ionospheric currents. The events typically caused a bipolar perturbation in the PC index. The first-negative-then-positive perturbation had an amplitude of about 0.5 in PC index and total duration of $10-30 \mathrm{~min}$. Using magnetic recordings from a network of observatories in the polar cap, Stauning and Troshichev (2008) inferred that the negative perturbation was related to the occurrence of a pair of transient dayside reverse (sunward in the central polar cap) convection vortices at cusp latitudes, caused by the divergence of magnetopause currents to the ionosphere. The more extended positive perturbation, on the other hand, was related to the formation of a pair of forward (antisunward in the central polar cap) convection vortices in the dayside oval, caused by enhanced Region 1 (R1) field-aligned currents. However, they did not separate their events according to northward or southward IMF orientation.

We begin by shortly describing the utilised instruments (Sect. 2). Section 3 presents the solar wind observations and an overview of the geomagnetic activity related to different parts of the sheath and cloud. Magnetospheric observations are described in Sect. 4, and ionospheric observations 
in Sect. 5. The final Sect. 6 contains discussion and conclusions.

\section{Instruments}

In this study we have combined measurements from several instruments observing the solar wind, the magnetosphere, and the ionosphere. Solar wind data are from the Advanced Composition Explorer (ACE), Solar and Heliospheric Observatory (SoHO), and Wind monitors, and magnetospheric observations from the Cluster, Double Star 1, and Geotail spacecraft. Several ground-based magnetometer arrays provided ionospheric data: $210^{\circ}$ Magnetic Meridian Circum-pan Pacific Magnetometer Network (210 MM CPMN, Yumoto and the CPMN Group, 2001) at $1 \mathrm{~s}$ resolution, CANadian Magnetic Observatory System (CANMOS, http://gsc.nrcan.gc.ca/geomag/obs/obsmap_e.php) at 5 s resolution, Canadian Array for Realtime Investigations of Magnetic Activity (CARISMA, Mann et al., 2008) at $1 \mathrm{~s}$ resolution, Geophysical Institute Magnetometer Array (GIMA, http://magnet.asf.alaska.edu/) at $1 \mathrm{~s}$ resolution, International Monitor for Auroral Geomagnetic Effects (IMAGE, http: //www.space.fmi.fi/image/) at $10 \mathrm{~s}$ resolution, Magnetometer Array for Cusp and Cleft Studies (MACCS, Hughes and Engebretson, 1997) at $5 \mathrm{~s}$ resolution, SuperMAG (http: //supermag.jhuapl.edu/index.html) at $1 \mathrm{~min}$ resolution, History of Events and Macroscale Interactions During Substorms (THEMIS, http://themis.ssl.berkeley.edu/) at $0.5 \mathrm{~s}$ resolution, and Troms $\varnothing$ Geophysical Observatory (TGO, http://geo.phys.uit.no/) at $10 \mathrm{~s}$ resolution. Super Dual Auroral Radar Network (SuperDARN, Chisham et al., 2007) observations have also been included. Moreover, several magnetic indices have been utilised: the $\mathrm{AE}$ and $\mathrm{PCN}$ indices at $1 \mathrm{~min}$ resolution and the Dst index (Sugiura, 1964) at $1 \mathrm{~h}$ resolution.

From the SoHO spacecraft we have utilised the results of the Shockspotter program (http://umtof.umd.edu/pm/FIGS. HTML), which attempts to identify possible interplanetary shocks using data from the Charge, Element, and Isotope Analysis System (CELIAS) Mass Time-Of-Flight (MTOF) Proton Monitor (PM) sensor (Hovestadt et al., 1995). From the ACE spacecraft we have used $16 \mathrm{~s}$ Level 2 data from the Magnetic Field Instrument (MAG, Smith et al., 1998) and 64 s Level 2 data from the Solar Wind Electron Proton Alpha Monitor (SWEPAM, McComas et al., 1998), and from the Wind spacecraft, $3 \mathrm{~s}$ (spin average) resolution data from the Magnetic Field Instrument (MFI, Lepping et al., 1995) and $3 \mathrm{~s}$ resolution ion moments from the 3DP instrument (Lin et al., 1995).

From the Cluster spacecraft, we have used both $4 \mathrm{~s}$ (spin average) and $0.04 \mathrm{~s}$ resolution data from the Fluxgate magnetometer (FGM, Balogh et al., 2001) and $4 \mathrm{~s}$ resolution data from Cluster Ion Spectrometry (CIS, Rème et al., 2001) Hot Ion Analyser (HIA) instrument. The high resolution mag-

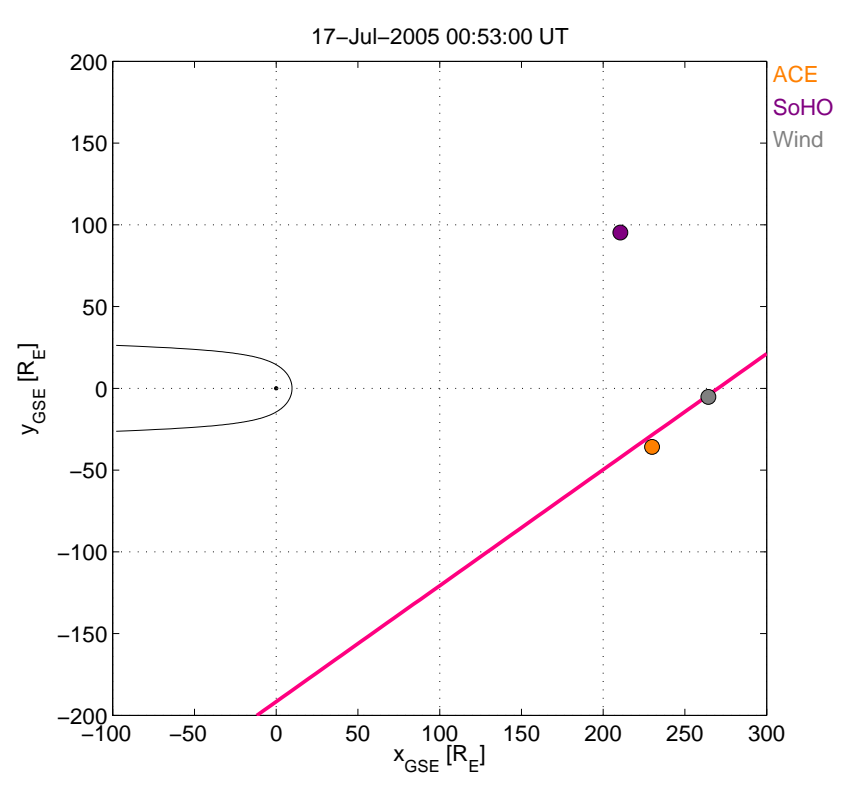

Fig. 1. Projections of the locations of the $\operatorname{ACE}\left((230,-36,23) R_{\mathrm{E}}\right)$, Wind $\left((264,-5,21) R_{\mathrm{E}}\right)$ and $\mathrm{SoHO}\left((210,95,6) R_{\mathrm{E}}\right)$ spacecraft to the xy-plane on 17 July 2005 at 00:53:00 UT. The leading edge of the sheath was observed by ACE at 00:51:25 UT, by Wind at 00:52:45 UT, and by SoHO at 01:23 UT. The direction and location of the leading edge, based on the timing analysis of these data, is illustrated by the red line. The black curve indicates the approximate magnetopause location.

netic field data have been utilised in minimum variance analysis (MVA), while the spin resolution data have been used for plotting. From Double Star 1, we have used $4 \mathrm{~s}$ (spin average) resolution data from FGM and 1 min summary parameters from HIA. From the Geotail spacecraft, we have utilised both $1 / 16 \mathrm{~s}$ resolution and $3 \mathrm{~s}$ resolution data from the Magnetic Field experiment (MGF, Kokubun et al., 1994), and $12 \mathrm{~s}$ resolution ion moments from the Low Energy Particle experiment (LEP, Mukai et al., 1994).

To present spacecraft data, we have used Cartesian $(x, y, z)$ Geocentric Solar Ecliptic (GSE) or Geocentric Solar Magnetospheric (GSM) coordinates, and to present ground-based data, spherical $(r, \theta, \phi)$ Geographic (GEO) or Solar Magnetic (SM) coordinates.

\section{Solar wind observations}

On 14 July 2005 at 11:20 UT, an x-ray flare accompanied by an asymmetric full halo CME was observed to occur on the Sun by SoHO. About $62 \mathrm{~h}$ later, on 17 July 2005 at 01:23 UT, the leading edge of the disturbance caused by the CME had reached SoHO, where it was observed as a discontinuity mainly in the solar wind speed and temperature (http://umtof.umd.edu/pm/FIGS.HTML).

The disturbance had already reached the ACE and Wind spacecraft about half an hour earlier. At 00:51:25 UT first 
ACE, and $1 \mathrm{~min} 20 \mathrm{~s}$ later, at 00:52:45 UT, also Wind observed a discontinuity in the parameters. Figure 1 displays projections of the locations of $\mathrm{ACE}\left(\operatorname{GSE}(230,-36,23) R_{\mathrm{E}}\right)$, SoHO $\left((210,95,6) R_{\mathrm{E}}\right)$ and Wind $\left((264,-5,21) R_{\mathrm{E}}\right)$ to the $\mathrm{xy}-$ plane on 17 July 2005 at 01:53 UT.

As ACE observed the discontinuity before Wind, but was located closer to Earth, it is clear that the discontinuity could not have been aligned in the y-direction, but must have been tilted, as illustrated by the red line in Fig. 1. This is in agreement with the flare observation: as the CME did not originate from the centre of the solar disk but from the right hand side edge as viewed from the Earth, the spacecraft would be expected to encounter the left hand side flank of the resulting ejecta.

Assuming that the discontinuity was planar, its alignment and speed could be determined from timing analysis (Paschmann and Daly, 2000) using the observation times and locations of ACE, Wind and SoHO. The resulting normal for the discontinuity was $(-0.58,0.82,0)$ and its speed in the direction of the normal $400 \mathrm{~km} / \mathrm{s}$. The zero z-component of the normal implies that the first contact of the front with the magnetopause took place at the equatorial plane.

Panels (a-f) in Fig. 2 display the IMF $\left(B_{\mathrm{x}}, B_{\mathrm{y}}, B_{\mathrm{z}}\right)$, $\mathrm{x}-$ component of the solar wind velocity $\left(V_{\mathrm{x}}\right)$, density $(N)$, and temperature $(T)$ measured by ACE and Wind from 16 July 2005 18:00:00 UT to 18 July 2005 12:00:00 UT. The first vertical magenta line in the figure denotes the discontinuity (17 July 2005 00:53 UT). At this time, $B_{\mathrm{x}}$ decreased from about -5 to $-7 \mathrm{nT}, B_{\mathrm{y}}$ was small and decreased from zero to about $-2 \mathrm{nT}$, and $B_{\mathrm{Z}}$ remained positive, but increased from about 2 to $7 \mathrm{nT}$, resulting in an increase of the total magnetic field $B$ from about 5 to $10 \mathrm{nT}$. The solar wind density $N$ increased from about 8 to $13 \mathrm{~cm}^{-3}$, and also the temperature increased. $V_{\mathrm{x}}$ decreased from -430 to $-460 \mathrm{~km} / \mathrm{s}$. The changes in $N$ and $V$ resulted in the doubling of the solar wind dynamic pressure from an initial value of about 3 to $6 \mathrm{nPa}$. The increase in $V, N, T$ and $B$ indicate a fast forward shock (Burlaga, 1971).

According to the Magnetic Cloud List at http://lepmfi.gsfc. nasa.gov/mfi/mag_cloud_pub1.html, a magnetic cloud was observed by Wind between 17 July 2005 15:18 UT (the second vertical magenta line) and 18 July 2005 03:48 UT (the third vertical magenta line). Panel (c) of Fig. 2 shows a firstpositive-then-negative bipolar $B_{\mathrm{Z}}$ signature, indicating that the orientation of the cloud was north-to-south. The shock observed by ACE, Wind and SoHO would therefore correspond to the leading edge of the sheath in front of the cloud.

The Dst index is displayed in panel (h) of Fig. 2. The horizontal lines in the plot lie at $0,-30$, and $-50 \mathrm{nT}$. According to Gonzalez et al. (1994), $-50 \mathrm{nT}<$ Dst $<-30 \mathrm{nT}$ indicates a weak magnetic storm, $-100 \mathrm{nT}<$ Dst $<-50 \mathrm{nT}$ a moderate storm, and Dst $<-100 \mathrm{nT}$ a strong storm. During several hours preceding the arrival of the sheath, the conditions were fairly quiet with Dst almost zero. The increase in the dynamic pressure related to the leading edge of the sheath caused a weak positive disturbance, and later during the passage of the sheath, an interval of strong negative $B_{\mathrm{Z}}$ caused a weak magnetic storm. A moderate storm was associated with the negative $B_{\mathrm{Z}}$ during the trailing part of the magnetic cloud.

The AE indices are shown in panel (g) of Fig. 2. Again, the effect of IMF $B_{\mathrm{Z}}$ was most obvious, as periods of negative $B_{\mathrm{Z}}$ produced activity, while during positive $B_{\mathrm{Z}}$ it was more quiet. At this resolution, it seems that there were not any obvious signatures related to the arrival of the leading edge of the sheath, and apart from periods of negative IMF $B_{\mathrm{z}}$, the sheath did not produce much activity. However, particularly during the trailing half of the magnetic cloud, when IMF $B_{\mathrm{Z}}$ was negative, there was more activity. In fact, the peak value of AE exceeded $1000 \mathrm{nT}$, indicating strong activity.

In the following sections, we will concentrate on the effects of the sudden dynamic pressure increase related to the arrival of the leading edge of the sheath on the magnetosphere-ionosphere system.

\section{Magnetospheric observations}

Figure 3 illustrates the locations of Cluster 1-4 (black $\quad(-9,-14,1) R_{\mathrm{E}}, \quad$ red $\quad(-10,-14,2) R_{\mathrm{E}}, \quad$ green $(-9,-13,1) R_{\mathrm{E}}$, blue $(-9,-13,1) R_{\mathrm{E}}$ ), Double Star 1 (pale red, $(-5,-11,4) R_{\mathrm{E}}$ ), and Geotail (pale blue, $(7,-7,1) R_{\mathrm{E}}$ ) spacecraft in the xy-plane on 17 July 2005 at 01:31 UT. The location of the leading edge of the sheath (red line) is based on the timing analysis (Sect. 3). The red arrow with tick marks illustrates its propagation, assuming that the velocity remained constant. The positions of the magnetopause (thick line) and bow shock (thin line) before (01:30 UT, black) and after (02:00 UT, grey) the pressure increase were given by the Grand Unified Ionosphere-Magnetosphere Coupling Simulation (GUMICS-4, Janhunen, 1996). As seen from the figure, the first contact of the oblique front with the magnetopause took place on the dawnside at about $(4,-14,0) R_{\mathrm{E}}$. The spacecraft were distributed along the outer edge of the dawnside magnetosphere, which made the configuration well suited for studying the effects of the front on the magnetopause location.

\subsection{Overview}

Figure 4 shows the satellite observations on 17 July 2005 between 01:20:00 and 02:00:00 UT. Panels (a-g) display the magnetic field $\left(B_{\mathrm{x}}, B_{\mathrm{y}}, B_{\mathrm{z}}\right)$, x-component of the plasma velocity $\left(V_{\mathrm{X}}\right)$, density $(N)$, and solar wind dynamic pressure as measured by ACE, Wind, Cluster, Double Star 1, and Geotail. The travel time of the planar front from the ACE and Wind locations to the magnetopause was given by

$\Delta t_{\left\{\begin{array}{c}\mathrm{ACE} \\ \text { Wind }\end{array}\right\}}=\frac{\left(\boldsymbol{r}_{\mathrm{mp}}-\boldsymbol{r}_{\left\{\begin{array}{l}\mathrm{ACE} \\ \text { Wind }\end{array}\right.}\right) \cdot \hat{\boldsymbol{n}}}{V}$, 


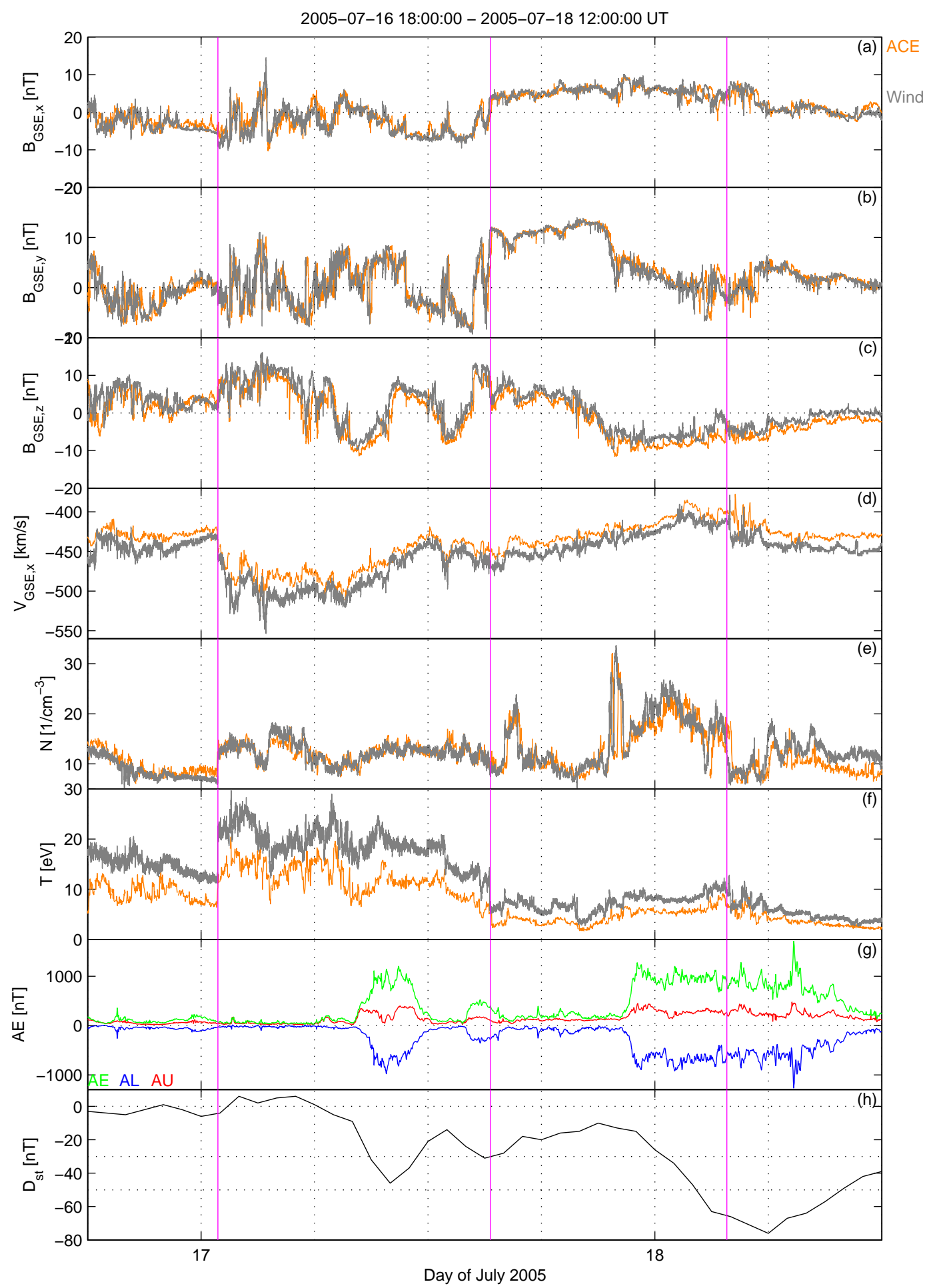

Fig. 2. Panels (a-f) interplanetary magnetic field $\left(B_{\mathrm{X}}, B_{\mathrm{y}}, B_{\mathrm{Z}}\right)$, x-component of the solar wind velocity $\left(V_{\mathrm{X}}\right)$, density $(N)$, and temperature $(T)$ measured by ACE and Wind from 16 July 2005 18:00:00 UT to 18 July 2005 12:00:00 UT. Panel (g) AE, AL, and AU indices. Panel (h) Dst index. The three vertical magenta lines denote the beginning of the sheath, the beginning of the magnetic cloud, and the end of the magnetic cloud. 


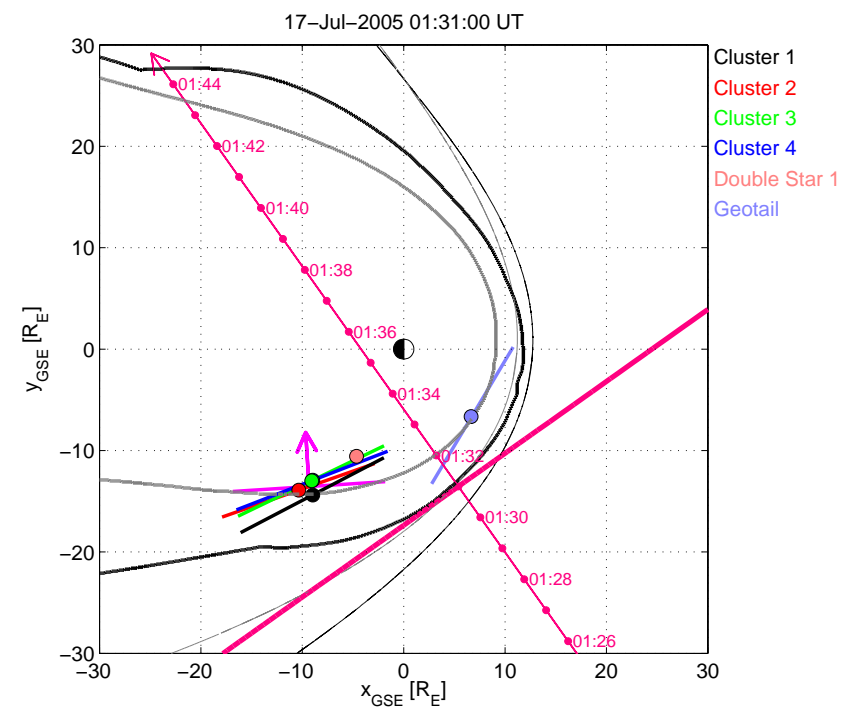

Fig. 3. Locations of Cluster $1-4$ (black $(-9,-14,1) R_{\mathrm{E}}$, red $(-10,-14,2) R_{\mathrm{E}}$, green $(-9,-13,1) R_{\mathrm{E}}$, blue $\left.(-9,-13,1) R_{\mathrm{E}}\right)$, Double Star 1 (pale red, $(-5,-11,4) R_{\mathrm{E}}$ ), and Geotail (pale blue, $\left.(7,-7,1) R_{\mathrm{E}}\right)$ spacecraft in the xy-plane on 17 July 2005 at 01:31 UT. The location of the leading edge of the sheath (red line) is based on the timing analysis (Sect. 3). The red arrow with tick marks illustrates its propagation, assuming that the velocity remained constant. The positions of the magnetopause (thick line) and bow shock (thin line) before (01:30 UT, black) and after (02:00 UT, grey) the pressure increase were given by the Grand Unified Ionosphere-Magnetosphere Coupling Simulation (GUMICS-4, Janhunen, 1996). The thick lines plotted with spacecraft colours display the alignment of the magnetopause according to minimum variance analysis (MVA) where it first passed each spacecraft, and the magenta line and arrow illustrate the orientation and direction of motion of the magnetopause according to timing analysis based on Cluster data.

where $\boldsymbol{r}_{\mathrm{mp}}=(4,-14,0) R_{\mathrm{E}}, \boldsymbol{r}_{\mathrm{ACE}}=(229.9,-35.8,22.7) R_{\mathrm{E}}$ and $\boldsymbol{r}_{\text {Wind }}=(264.4,-5.3,20.5) R_{\mathrm{E}}$, and $\hat{\boldsymbol{n}}$ and $V$ are the normal vector and speed of the front obtained from the timing analysis (Sect. 3). The resulting time delays from ACE and Wind were about 40 and $38 \mathrm{~min}$, respectively. The ACE and Wind data in Fig. 4 have been shifted accordingly.

At about 01:31 UT, the discontinuity was estimated to arrive at the magnetopause, and the solar wind dynamic pressure abruptly increased from about 3 to $6 \mathrm{nP}$. A few minutes later, the first effects were observed by the magnetospheric spacecraft. As a consequence of the compression of the magnetosphere, Geotail almost immediately moved from the magnetosphere to the magnetosheath: the magnetic field changed from fairly stable to strongly variable and plasma speed increased from below $100 \mathrm{~km} / \mathrm{s}$ to about $300 \mathrm{~km} / \mathrm{s}$, which was still lower than the solar wind speed. Plasma density increased from about 1 to $30 \mathrm{~cm}^{-3}$, after which it settled around $10 \mathrm{~cm}^{-3}$.
Also Cluster moved from the magnetosphere to the magnetosheath with the dynamic pressure increase: all magnetic field components became more variable, $B_{\mathrm{x}}$ decreased from about $20 \mathrm{nT}$ to slightly negative values, and $V$ and $N$ increased almost to the level of solar wind values. However, almost immediately Cluster 3 and Cluster 4 returned inside the magnetosphere. This is evident from $B_{\mathrm{X}}$ becoming again positive, and from the decreasing of $V$. For the next several hours, Cluster 3 and 4 were mainly located inside the magnetosphere, and Cluster 1 and 2 outside.

Unlike Geotail and Cluster, Double Star 1 did not exit the magnetosphere when the pressure front hit. The increase in dynamic pressure was merely evident in the enhancement of $B_{\mathrm{x}}$ and $B_{\mathrm{Z}}$.

Thus, the abrupt increase of dynamic pressure compressed the magnetosphere such that Cluster and Geotail shifted from the magnetosphere to the magnetosheath while Double Star 1 remained in the magnetosphere. Almost immediately this overshoot was corrected, and the magnetosphere expanded again so that the magnetopause ended up between the Cluster spacecraft with Cluster 1 and 2 in the magnetosheath and Cluster 3 and 4 in the magnetosphere. Next, we analyse the orientation and motion of the magnetopause after the pressure increase in more detail.

\subsection{Arrival of the pressure front}

The initial signs of the compression of the magnetosphere caused by the dynamic pressure increase can be seen in Fig. 4 as the enhancement of the magnetic field measured by the magnetospheric spacecraft. Geotail, located close to the subsolar point, was the first to observe the beginning of the compression at 01:34:10 UT. Cluster 1-4 and Double Star 1, located close to the magnetopause on the dawnside, were the next at 01:35:59 (Cluster 1), 01:36:10 (Cluster 2), 01:36:01 (Cluster 3), 01:36:01 (Cluster 4), and 01:36:00 UT (Double Star 1).

The inward moving magnetopause passed Geotail at 01:36:55 UT. According to the minimum variance analysis (MVA, Paschmann and Daly, 2000), the magnetopause normal was $(-0.84,0.50,-0.19)$. In Fig. 3, the orientation of the magnetopause at Geotail is represented by the pale blue line. Similarly, the inward moving magnetopause passed Cluster 1-4, for the first time at 01:36:56 UT, 01:37:31 UT, 01:39:00 UT, and 01:38:59UT. The magnetopause normals given by MVA were $(0.46,-0.88,0.08),(0.33,-0.94,0.08)$, $(0.43,-0.90,-0.05)$, and $(0.36,-0.93,-0.04)$, and the resulting magnetopause tangents have been marked at each Cluster location in Fig. 3. All magnetopause tangents in the figure agree with the GUMICS magnetopause direction.

The close proximity of the Cluster spacecraft also provided another means of approximating the orientation as well as the velocity of the magnetopause: timing analysis. The resulting magnetopause normal $(-0.06,0.94,-0.34)$ was in agreement with the MVA results, and the speed of the 


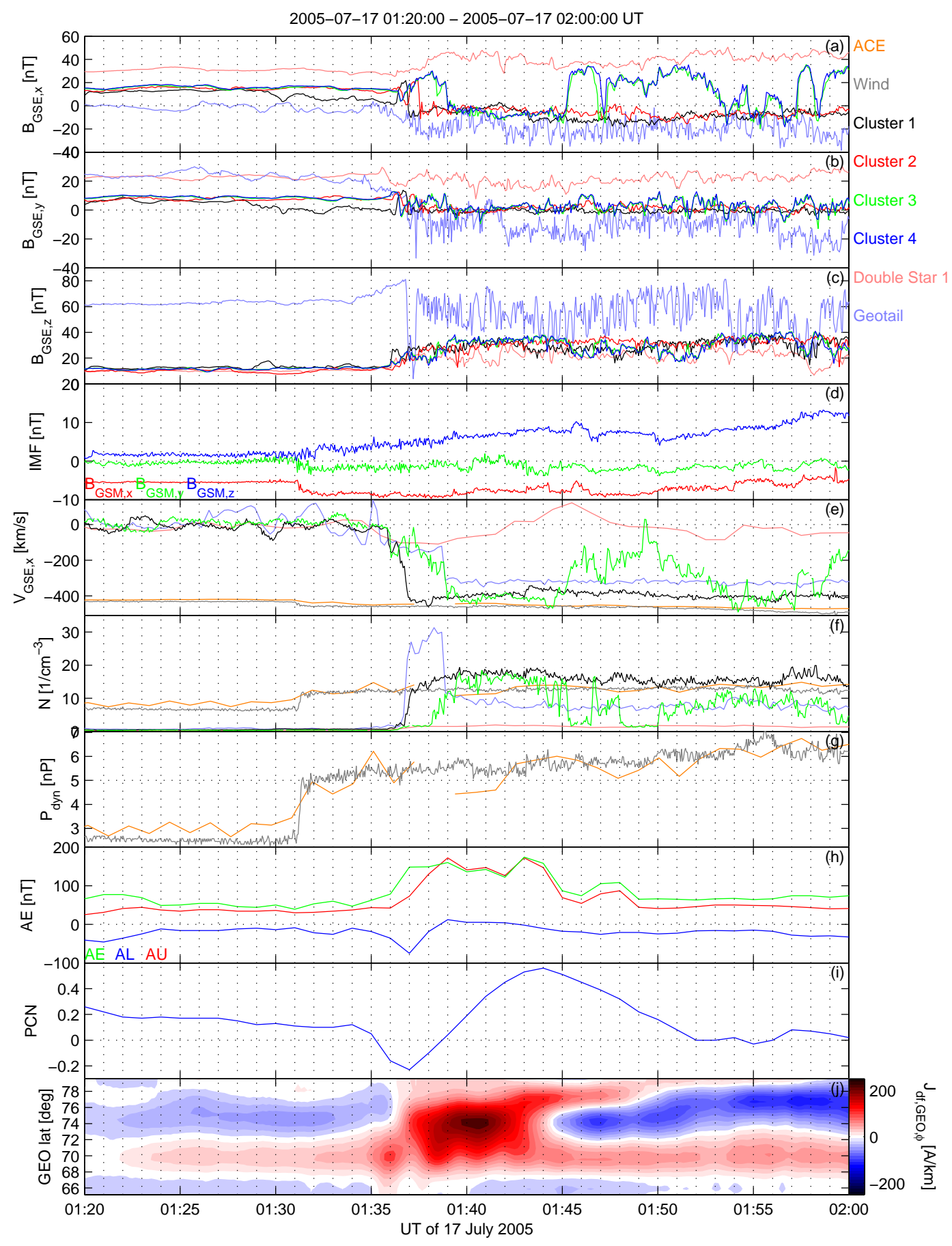

Fig. 4. Panels (a-c) magnetic field $\left(B_{\mathrm{X}}, B_{\mathrm{y}}, B_{\mathrm{Z}}\right)$ as measured by Cluster, Double Star 1, and Geotail on 17 July 2005 between 01:20:00 and 02:00:00 UT. Panel (d) Three GSM components of the IMF as measured by Wind. Panels (e-f) x-component of plasma velocity ( $V_{\mathrm{x}}$ ) and density $(N)$ as measured by ACE, Wind, Cluster 1 and Cluster 3, Double Star 1, and Geotail. Panel (g) solar wind dynamic pressure as measured by ACE and Wind. ACE and Wind data have been time-delayed by about 40 and 38 min, respectively, so that they would approximately correspond to observations at the magnetopause at the given time. Panel (h) AE, AL, and AU indices. Panel (i) PCN index. Panel (j) one-dimensional ionospheric equivalent current density in the geographic (GEO) east-west direction $\left(J_{\mathrm{GEO}, \phi}\right)$ as a function of geographic latitude, determined from the magnetic field data of the IMAGE TAR-NAL chain (blue squares in Fig. 6a). Eastward currents are shown with a positive and westward currents with a negative sign. 


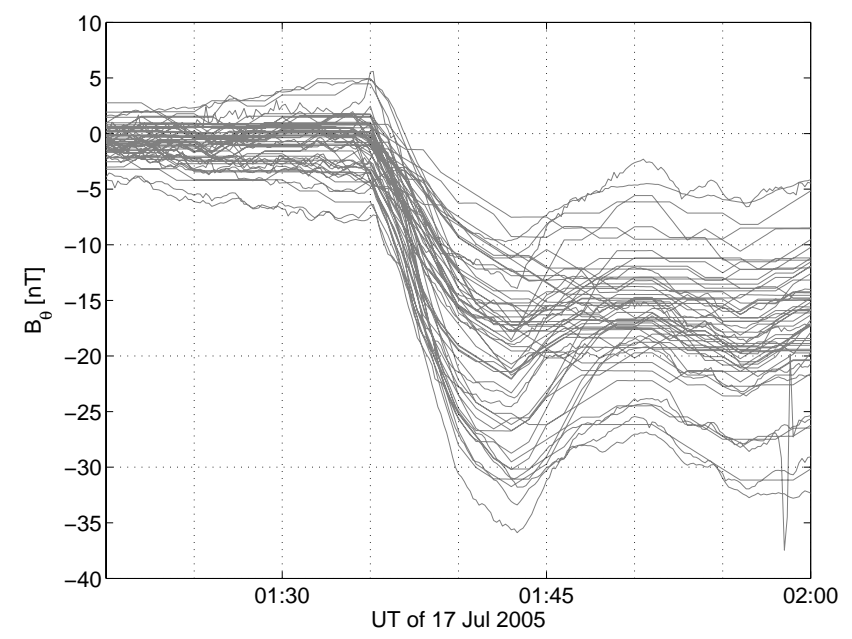

Fig. 5. Variation of the $\operatorname{SM} \theta$ component of the magnetic field measured by 65 low latitude (SM $|1 \mathrm{lat}|<45^{\circ}$ ) SuperMAG magnetometers on 17 July 2005 between 01:20 and 02:00 UT. The magnetometer locations spanned all local time sectors.

magnetopause was $60 \mathrm{~km} / \mathrm{s}$. The magenta line and arrow in the middle of the Cluster constellation in Fig. 3 represent the orientation and direction of motion of the magnetopause according to the timing analysis. Assuming that the magnetopause started moving slightly after 01:31 UT, it took it about $5 \mathrm{~min}$ to reach the location of Cluster 1. Based on Fig. 3, the distance can roughly be estimated to be about $5 R_{\mathrm{E}}$. This would correspond to an average velocity of about $100 \mathrm{~km} / \mathrm{s}$.

The inward moving dawnside magnetopause passed Geotail and Cluster between 01:37 and 01:39UT. Assuming that the location of the leading edge of the sheath marked in Fig. 3 is approximately correct, by this time the front was already well past Earth and the new equilibrium position of the dawnside magnetopause. The dawnside magnetopause position stabilised and settled between the Cluster spacecraft at about 01:45 UT. Thus, the magnetopause was moving for about $15 \mathrm{~min}$ after first contact with the front of dynamic pressure increase.

\section{Ionospheric observations}

\subsection{Low latitude signatures}

Figure 5 displays the variation of the $\operatorname{SM} \theta$ component of the magnetic field measured by 65 low latitude (SM $\mid$ lat $\mid<45^{\circ}$ ) SuperMAG magnetometers between 01:20 and 02:00 UT. The magnetometer locations spanned all local time sectors.

$B_{\theta}$ began to decrease at about 01:34 UT, and the minimum was reached at 01:43 UT. Between 01:43 and 01:50 UT, $B_{\theta}$ again increased slightly. This behaviour is in agreement with the observed magnetopause motion: the magnetopause was estimated to have started moving earthward soon af- ter 01:31 UT, at 01:34 UT first signatures were observed by Geotail, and between 01:37 and 01:39 UT the magnetopause passed Geotail and Cluster. At 01:43 UT the magnetic field measured by Double Star 1 peaked, indicating that the magnetopause had reached its earthwardmost point and started expanding again. Approximately at 01:45 UT, the magnetopause returned in the middle of the Cluster constellation and settled there. The small decrease between about 01:50 and 02:00 UT corresponds to the small increase in dynamic pressure (panel g of Fig. 4). During this time, the magnetopause also moved a little earthward and then back again, such that Cluster 3 and 4 moved from the magnetosphere to the magnetosheath and back. However, the variations of $B_{\mathrm{x}}$ during this time (panel a of Fig. 4) indicate that the spacecraft remained close to the magnetopause.

\subsection{High latitude signatures}

\subsubsection{1-D view}

Panel (h) of Fig. 4 shows the AE, AL, and AU indices. The decrease of AL observed between about 01:34 and 01:38 UT was quite small, but AU displayed a clear increase between about 01:36 and 01:49UT. After the increase of dynamic pressure, AE was slightly higher than during lower pressure, indicating that the ionospheric equivalent currents were more intense.

Panel (i) of Fig. 4 shows the PC index. The index displayed the typical bipolar signature associated with a pressure pulse (Stauning and Troshichev, 2008), consisting of a negative perturbation between about 01:34 and 01:39 UT followed by a longer and stronger positive perturbation between about 01:39 and 01:52 UT. Altogether the signature lasted $18 \mathrm{~min}$.

Panel (j) of Fig. 4 displays the one-dimensional ionospheric equivalent current density in GEO east-west direction ( $J_{\mathrm{GEO}, \phi}$, Vanhamäki et al., 2003) as a function of GEO latitude, determined from the magnetic field data of the IMAGE TAR-NAL chain (blue squares in Fig. 6a). Eastward equivalent current density is shown with a positive and westward with a negative sign. As the displayed time interval from 01:20 to 02:00 UT corresponds to approximately 04 MLT, the dominant feature expected in the plots would be the westward electrojet. It would be stronger and located more equatorward during negative IMF $B_{\mathrm{Z}}$ conditions, and weaker and located more poleward during positive IMF $B_{\mathrm{z}}$ conditions (e.g., Anderson et al., 2008).

The half an hour interval preceding the arrival of the leading edge of the sheath at about 01:31 UT was very quiet due to the prevailing weak northward IMF conditions (panel $\mathrm{h}$ in Fig. 4). The westward (blue) electrojet, although very weak, was located around $75^{\circ}$ latitude. Equatorward of the electrojet, around $70^{\circ}$ latitude, there was weak eastward (red) equivalent current. One minute after the predicted arrival of the pressure pulse, at 01:32 UT, the westward electrojet 

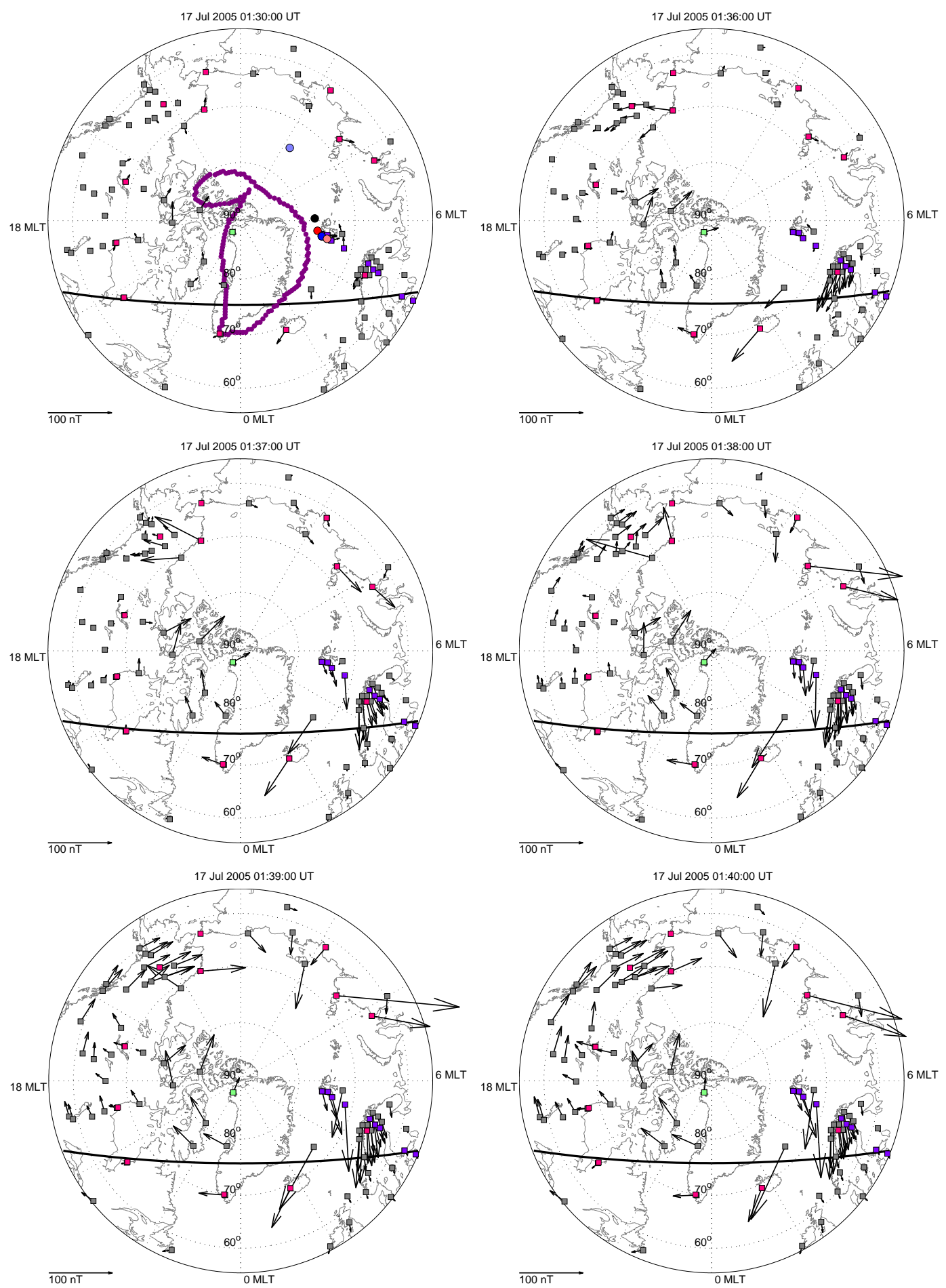

Fig. 6a. Magnetic field observations from the $210 \mathrm{MM}$ CPMN, CANMOS, CARISMA, GIMA, IMAGE, MACCS, SuperMAG, TGO, and THEMIS magnetometer arrays on 17 July 2005 at 01:30, 01:36, 01:37, 01:38, 01:39, 01:40, 01:42, 01:44, 01:46, 01:48, 01:50, and 02:00 UT. The horizontal magnetic field vectors, displayed by the black arrows, have been rotated $90^{\circ}$ anticlockwise to obtain a proxy for the ionospheric $\boldsymbol{E} \times \boldsymbol{B}$ drift. The magnetometer locations are indicated by the squares, with the stations of the IMAGE TAR-NAL chain highlighted in blue, the AE stations in red, and the Northern Hemisphere PC station in green. The black curve represents the terminator. The approximate Cluster, Double Star 1, and Geotail footprints, and the open-closed field line boundary (purple dots) before (01:30 UT panel) and after (02:00 UT panel) the pressure increase were given by the GUMICS simulation. 

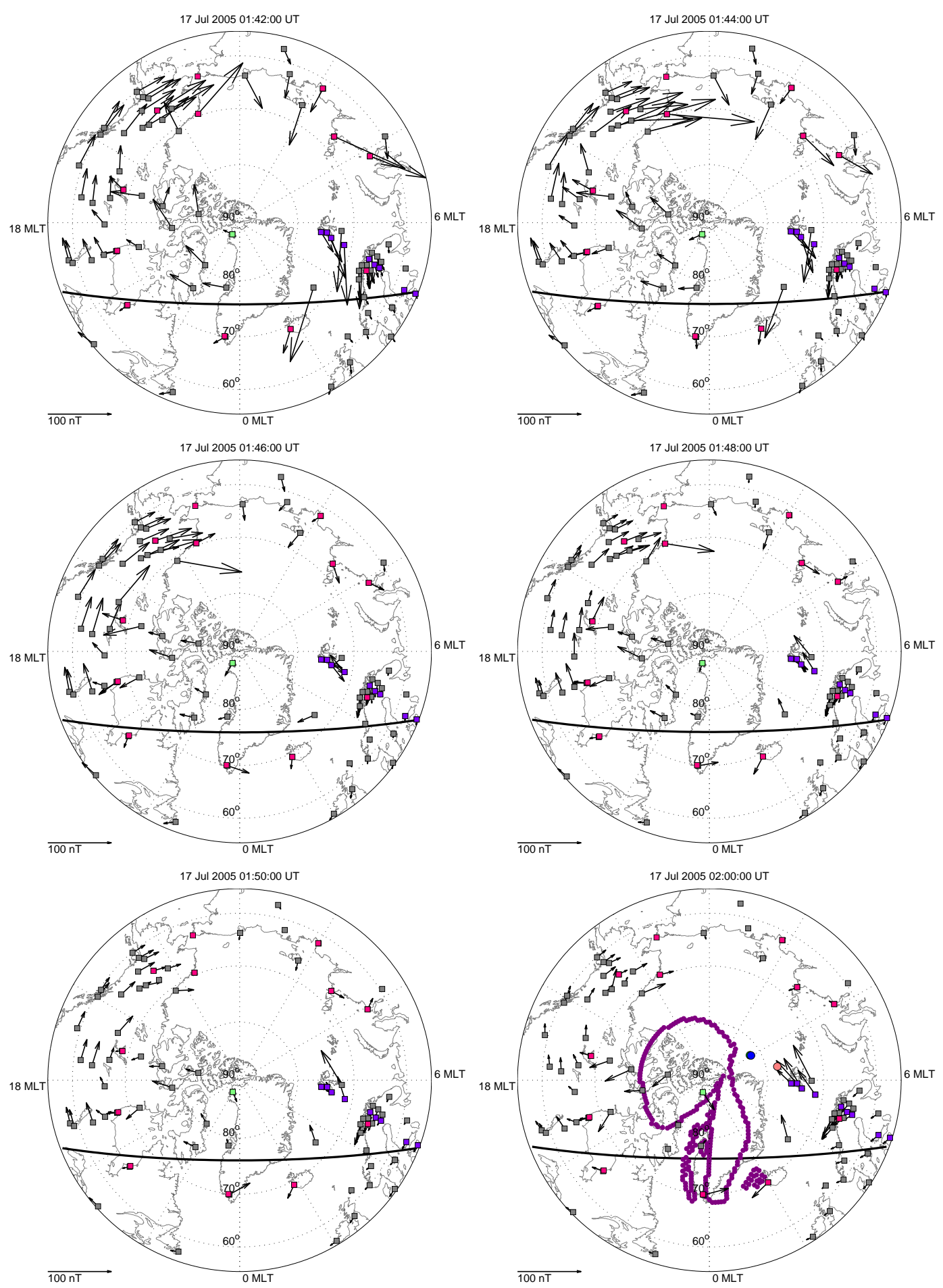

Fig. 6b. Continued.

and the poleward boundary of the eastward equivalent current started to shift poleward. Between about 01:34 and 01:37 UT, the eastward current around $70^{\circ}$ latitude was intensified. Around 01:37 UT, a second, stronger intensification appeared, extending from about $68^{\circ}$ to $78^{\circ}$ latitude. The maximum current intensity was reached around 01:41 UT, af- ter which the eastward equivalent current began to weaken. At about 01:45 UT, a region of westward equivalent current appeared around $74^{\circ}$ latitude, and the eastward equivalent current was deflected poleward and equatorward of this region. 
Consistent with previous observations, the signatures in AE, PC and $J_{\mathrm{GEO}, \phi}$ all appeared to consist of two parts: the shorter and less intense preliminary part between about 01:34 and 01:37 UT comprised the decrease of AL, the decrease of PC, and the first, fainter, increase of eastward equivalent current. The longer and more intense main part of the signature between about 01:37 and 01:45 UT, on the other hand, comprised the increase of $\mathrm{AU}$, the increase of PC, and the second, latitudinally more extensive, increase of the eastward equivalent current.

Compared to magnetospheric satellite data, the increase in $\mathrm{AU}, \mathrm{PC}$, and $J_{\mathrm{GEO}, \phi}$ between 01:37 and 01:43 UT coincided with the inward motion of the dawnside magnetopause observed by the spacecraft. However, as discussed in the previous section, the motion of the dawnside magnetopause was likely to have began already before this, soon after the pressure front impact at 01:31 UT. At about 01:43 UT, the enhancement of the magnetic field observed by Double Star 1 started to decrease, and a few minutes later, around 01:45 UT, Cluster 3 and Cluster 4 returned from the magnetosheath to the magnetosphere. Therefore, the weakening of AU, PC and $J_{\mathrm{GEO}, \phi}$ appears to have coincided with the beginning of the outward motion of the magnetopause.

Thus, the most obvious high-latitude ionospheric signatures associated with the dynamic pressure increase during northward IMF conditions seemed to be related to the changes in the magnetopause position. As the position stabilised, these signatures also disappeared.

\subsubsection{2-D view}

Figures $6 \mathrm{a}$ and $6 \mathrm{~b}$ show snap shots of the magnetic field observations from the 210 MM CPMN, CANMOS, CARISMA, GIMA, IMAGE, MACCS, SuperMAG, THEMIS and TGO magnetometer arrays between 01:30 and 02:00 UT. The horizontal magnetic field vectors, displayed by the black arrows, have been rotated $90^{\circ}$ anticlockwise to obtain a proxy for the ionospheric $\boldsymbol{E} \times \boldsymbol{B}$ drift. The magnetometer locations have been indicated by the squares, with the stations of the IMAGE TAR-NAL chain highlighted in blue, the AE stations in red, and the Northern Hemisphere PC station in green. The black curve represents the terminator. The approximate Cluster, Double Star 1, and Geotail footprints, and the open-closed field line boundary (purple dots) before (01:30 UT panel) and after (02:00 UT panel) the pressure increase were given by the GUMICS simulation. The open-closed field line boundaries correspond to the two magnetopause locations in Fig. 3. The boundary was more compressed on the dawnside after the pressure increase than before it, while the duskside was more round. This change could have been produced by lobe reconnection that resulted from the IMF $B_{\mathrm{y}}$ sign flip, as shown by panel (d) in Fig. 4. Also the irregular shape of the open field line regions could have been caused by the magnetotail twisting due to the prevailing IMF conditions (Kullen and Janhunen, 2004). Geo- tail, Cluster 1 and Cluster 2 moved from the magnetosphere to the magnetosheath after the pressure pulse, and hence they do not have any ionospheric footprints in the 02:00 UT panel. Due to the reconfiguration of the magnetosphere, the footprints of Cluster 3, Cluster 4, and Double Star 1 were shifted from their original positions poleward and towards the dayside. As yet, GUMICS-4 does not support oblique discontinuities, which is why we have only included the open field line polar cap boundary locations well before and after the arrival of the front. Although the corresponding magnetopause positions coincided very well with observations, what happened between did not, because the geometry of the arriving front was not correct.

The top left panel of Fig. 6a displays the equivalent convection pattern at 01:30 UT, before the pressure increase. The convection was very weak and the polar cap small. Just outside the polar cap the convection pattern indicated the typical auroral oval configuration, with eastward convection on the danwside and westward convection on the duskside. At lower latitudes the convection was mainly westward both at the dawn and dusk sectors.

The first clear signatures related to the dynamic pressure increase appeared at 01:36 UT (top right). The pre-existing westward convection intensified, and a region of eastward (antisunward) convection appeared around 14 MLT. The pattern is consistent with that of a pair of reverse convection vortices (e.g., Stauning and Troshichev, 2008). The symmetry axis of the pattern appears to have been tilted from noon to about 09 MLT, consistent with the oblique pressure front.

One minute later, at 01:37 UT (middle left), westward (antisunward) convection had appeared around 09 MLT, and also convection at the IMAGE (03-06 MLT) high latitude stations had turned from eastward (sunward) to westward (antisunward). Around 14 MLT, the eastward (antisunward) convection started to turn westward (sunward). By 01:40 UT (middle right), convection had turned mainly westward everywhere, forming a vortex encircling possibly the magnetic pole or cusp region.

The panels of Fig. $6 \mathrm{~b}$ illustrate how the convection weakened and returned back to normal configuration. Around 19 MLT, convection turned from westward to southward (antisunward). Starting at midnight (the station at the southern tip of Greenland) and proceeding towards noon on the dawnside, convection at high latitudes turned from westward (antisunward) to eastward (sunward). By 02:00 UT (bottom right), the configuration that existed before the pressure increase had re-emerged, but with slightly enhanced convection.

The eastward turning of the convection around 14 MLT between 01:36 and 01:38 UT corresponded to the decrease of AL, that is, the preliminary signature. Between 01:39 and 01:43 UT all convection was more or less westward, explaining why AL was zero but AU enhanced. These observations are in agreement with those of Iyemori and Araki (1982). The recovery of the eastward convection on the 


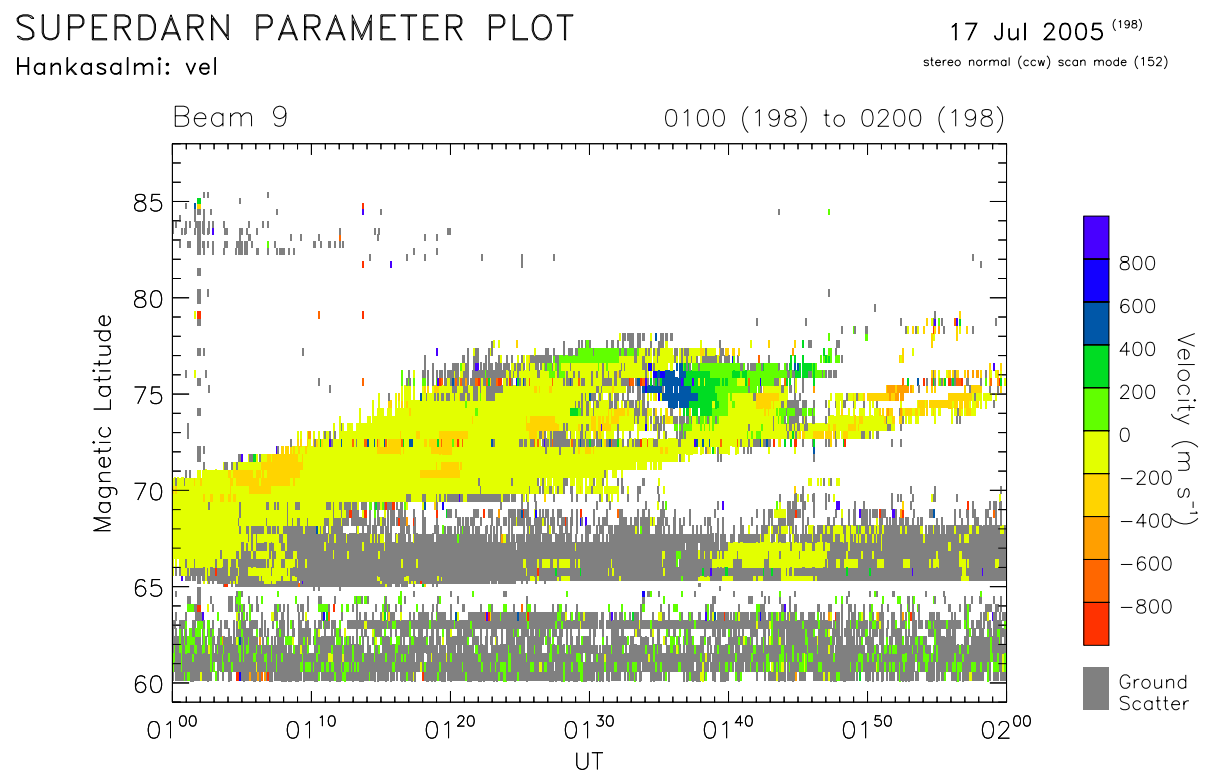

Fig. 7. Line-of-sight velocity from Beam 9 of the Hankasalmi SuperDARN radar on 17 July 2005 between 01:00 and 02:00 UT. Line-of-sight component of flow towards the radar along the beam is plotted with a positive sign (blue and green colours) and away from the radar with a negative sign (yellow and red colours).

dawnside was visible as a small negative signature in $\mathrm{AL}$ from 01:44 UT onwards.

\subsubsection{CUTLASS data}

The Co-operative UK Twin Auroral Sounding System (CUTLASS, http://ion.le.ac.uk/cutlass/cutlass.html) forms part of the SuperDARN network of radars. It consists of two HF coherent radars, one at Hankasalmi, Finland, and one at Pykkvibær, Iceland. Each radar measures the line-of-sight velocity component of the ionospheric $\boldsymbol{E} \times \boldsymbol{B}$ drift, but the overlapping field-of-views of the two radars allows the measurements to be combined as full 2-D velocity vectors. The Pykkvibær radar monitors the approximately east-west component of the $\boldsymbol{E} \times \boldsymbol{B}$ drift in the IMAGE region, while the Hankasalmi radar monitors the north-south component.

Unfortunately during our event, there was very little backscatter from the Pykkvibær radar. As the magnetic field data suggested mainly changes in the east-west velocity component, this made comparing the two measurements more complicated. Moreover, the Hankasalmi backscatter originated mainly slightly north of the IMAGE field-of-view. Nonetheless, the Hankasalmi radar did show some effect.

Figure 7 displays the line-of-sight velocity from Beam 9 of the Hankasalmi radar between 01:00 and 02:00 UT. During the displayed time interval, the region contributing backscatter moved poleward, indicating that the polar cap was contracting in agreement with the prevailing northward IMF conditions. Between about 01:34 and 01:44 UT, the data showed positive line-of-sight Doppler shifts, which indicate a significant southward component to the flow. The existence of this signature coincided well with the eastward equivalent signature in panel (j) of Fig. 4 and the convection pattern in Fig. 6a and $6 \mathrm{~b}$.

\section{Discussion and conclusions}

In this study we have traced the effects produced by a CME from the solar wind to Earth's magnetosphere and ionosphere. In the solar wind, an earthward bound north-south oriented magnetic cloud and its sheath were observed by the ACE, SoHO, and Wind spacecraft. A steplike increase of the solar wind dynamic pressure during northward IMF conditions was related to the leading edge of the sheath. A timing analysis between the three spacecraft revealed that the front was not aligned with the GSE y-axis, but had a normal $(-0.58,0.82,0)$. A tilt this big significantly affects the delay time between the spacecraft and the magnetosphere. Knowing the exact arrival time of different solar wind structures is essential when studying their effect on the magnetosphereionosphere system.

Due to the orientation of the leading edge of the sheath, its first contact with the magnetosphere occurred on the dawnside at about $(4,-14,0) R_{\mathrm{E}}$. Fortunately, Cluster, Double Star 1, and Geotail happened to be distributed close to the magnetopause in this region, which made it possible to closely monitor the motion of the magnetopause. The inward motion of the magnetopause due to the enhancement of the dynamic pressure moved Cluster and Geotail almost immediately from the magnetosphere to the magnetosheath. Double Star 1 remained inside the magnetosphere. When 
the pressure pulse impacted the magnetosphere, the magnetopause was first perceived to move inward and then immediately correct the overshoot by slightly expanding again such that it ended up between the Cluster constellation with Double Star 1 inside the magnetosphere and Geotail in the magnetosheath.

Coinciding with the inward and subsequent outward motion of the magnetopause, the ground-based magnetic field at low latitudes was observed to first strengthen and then weaken. As the magnetopause position stabilised, so did the ground-based magnetic field intensity, settling at a level slightly higher than before the pressure increase. Altogether the magnetopause was moving for about $15 \mathrm{~min}$ after first contact with the front.

The high latitude ionospheric signature consisted of two parts: a shorter (few minutes) and less intense preliminary part comprised a decrease of AL and a negative variation of PC. A longer (about ten minutes) and more intense main part of the signature comprised an increase of $\mathrm{AU}$ and a positive variation of $\mathrm{PC}$.

A proxy for the ionospheric $\boldsymbol{E} \times \boldsymbol{B}$ drift was obtained by rotating horizontal ground-based magnetic field vectors $90^{\circ}$ anticlockwise. Before the pressure increase, a configuration typical for the prevailing northward IMF conditions was observed at high latitudes. The preliminary signature coincided with a pair of reverse convection vortices, whereas during the main signature, mainly westward convection was observed at all local time sectors. Afterwards, the configuration preceding the pressure increase was recovered, but with slightly enhanced convection.

The magnetospheric and ionospheric effects followed the calculated arrival of the pressure increase within a few minutes, confirming that the delay time due to the oblique front was accurate within a few minutes resolution. The timing analysis that was used to determine the arrival time of the front requires at least three spacecraft in the solar wind observing the same signature. We used ACE, SoHO, and Wind, but if either Cluster 1-4, THEMIS 1-5 or Geotail happens to be in the solar wind, it could be used instead of SoHO that is not equipped with a magnetometer. Based on the timing analysis (Fig. 3), the existence of the preliminary signature (01:34-01:37 UT) coincided with passage of the oblique pressure front, whereas during the main signature (01:3701:45 UT), the front was already well past the Earth. The main signature existed during the time the magnetopause was observed to move. As the position stabilised, also the signature disappeared.

The observation of the pair of reverse convection vortices during the preliminary signature is in agreement with earlier findings (e.g., Stauning and Troshichev, 2008). However, Stauning and Troshichev (2008) associated the main signature with the formation of a pair of forward convection vortices, while we observed mainly westward convection at all local time sectors. In our case, the normal convection configuration was resumed later, after the main signature had disappeared. However, Stauning and Troshichev (2008) did not separate their data according to northward or southward IMF conditions, which could explain the difference. Iyemori and Araki (1982), on the other hand, studied dynamic pressure variations specifically under northward IMF conditions, and observed the single convection vortex during the main signature.

Acknowledgements. The IMAGE magnetometer data are collected as an Estonian-Finnish-German-Norwegian-Polish-RussianSwedish project. We acknowledge the principal investigators D. J. McComas (ACE/SWEPAM), N. Ness (ACE/MAG), E. Lucek (Cluster/FGM), I. Dandouras (Cluster/CIS), C. Carr (Double Star 1/FGM), H. Réme (Double Star 1/HIA), T. Nagai (Geotail/MGF), Y. Saito (Geotail/LEP), R. Lepping (Wind/MFI), and R. Lin (Wind/3DP). The spacecraft data were acquired through CDAWeb, Cluster Active Archive, and DARTS at Institute of Space and Astronautical Science, JAXA in Japan. We thank T. Zhang for providing the Double Star FGM data. We acknowledge the principal investigators K. Yumoto (210 MM CPMN), Natural Resources Canada (CANMOS), I. R. Mann (CARISMA), M. Engebretson and W. J. Hughes (MACCS), S. Mende and C. T. Russell (THEMIS GMAG), and J. W. Gjerloev and contributing PIs (SuperMAG). 210 MM CPMN stations are maintained by the Space Environment Research Center, Kyushu University, CANMOS by the Geological Survey of Canada, CARISMA by the University of Alberta, funded by the Canadian Space Agency, GIMA by the Geophysical Institute, University of Alaska, Fairbanks, and MACCS by Boston University and Augsburg College. We acknowledge NASA contract NAS5-02099 and V. Angelopoulos for use of data from the THEMIS Mission. We thank Troms $\varnothing$ Geophysical Observatory (TGO) for the use of Jan Mayen magnetic field data. CANMOS and CARISMA data were acquired through CCSDP and THEMIS data through CDAWeb. We thank K. Shiokawa for distributing the 210 MM CPMN data and I. Honkonen for running the GUMICS simulation. The work of L. Juusola was supported by the Academy of Finland. P. Stauning and an anonymous referee are thanked for their contribution.

Topical Editor I. A. Daglis thanks P. Stauning and another anonymous referee for their help in evaluating this paper.

\section{References}

Amm, O., Engebretson, M. J., Hughes, T., Newitt, L., Viljanen, A., and Watermann, J.: A traveling convection vortex event study: Instantaneous ionospheric equivalent currents, estimation of field-aligned currents, and the role of induced currents, J. Geophys. Res., 107(A11), 1334, doi:10.1029/2002JA009472, 2002.

Anderson, B. J., Korth, H., Waters, C. L., Green, D. L., and Stauning, P.: Statistical Birkeland current distributions from magnetic field observations by the Iridium constellation, Ann. Geophys., 26, 671-687, doi:10.5194/angeo-26-671-2008, 2008.

Balogh, A., Carr, C. M., Acuña, M. H., Dunlop, M. W., Beek, T. J., Brown, P., Fornacon, K.-H., Georgescu, E., Glassmeier, K.H., Harris, J., Musmann, G., Oddy, T., and Schwingenschuh, K.: The Cluster Magnetic Field Investigation: overview of in-flight performance and initial results, Ann. Geophys., 19, 1207-1217, doi:10.5194/angeo-19-1207-2001, 2001. 
Burlaga, L., Sittler, E., Mariani, F., and Schwenn, R.: Magnetic Loop Behind an Interplanetary Shock: Voyager, Helios, and IMP 8 Observations, J. Geophys. Res., 86(A8), 6673-6684, 1981.

Burlaga, L. F.: Hydromagnetic Waves and Discontinuities in the Solar Wind, Space Sci. Rev., 12, 600-657, 1971.

Chisham, G., Lester, M., Milan, S. E., Freeman, M. P., Bristow, W. A., Grocott, A., McWilliams, K. A., Ruohoniemi, J. M., Yeoman, T. K., Dyson, P. L., Greenwald, R. A., Kikuchi, T., Pinnock, M., Rash, J. P. S., Sato, N., Sofko, G. J., Villain, J.P., and Walker, A. D. M.: A decade of the Super Dual Auroral Radar Network (SuperDARN): Scientific achievements, new techniques and future directions, Surv. Geophys., 28, 33-109, doi:10.1007/s10712-007-9017-8, 2007.

Davis, T. N. and Sugiura, M.: Auroral electrojet activity index AE and its universal time variations, J. Geophys. Res., 71, 785, 1966.

Fukushima, N.: Generalized theorem for no ground magnetic effect of vertical currents connected with Pedersen currents in the uniform-conductivity ionosphere, Rep. Ionos. Space Res. Japan, 30, 35-40, 1976.

Glassmeier, K.-H.: Traveling magnetospheric convection twinvortices - Observations and theory, Ann. Geophys., 10, 547-565, 1992.

Gonzalez, W. D., Joselyn, J. A., Kamide, Y., Kroehl, H. W., Rostoker, G., Tsurutani, B. T., and Vasyliunas, V. M.: What is a Geomagnetic Storm?, J. Geophys. Res., 99(A4), 5771-5792, 1994.

Hovestadt, D., Hilchenbach, M., Burgi, A., Klecker, B., Laeverenz, P., Scholer, M., Grunwaldt, H., Axford, W. I., Livi, S., Marsch, E., Wilken, B., Winterhoff, H. P., Ipavich, F. M., Bedini, P., Coplan, M. A., Galvin, A. B., Gloeckler, G., Bochsler, P., Balsiger, H., Fischer, J., Geiss, J., Kallenbach, R., Wurz, P., Reiche, K.-U., Gliem, F., Judge, D. L., Ogawa, H. S., Hsieh, K. C., Mobius, E., Lee, M. A., Managadze, G. G., Verigin, M. I., and Neugebauer, M.: CELIAS - The Charge, Element and Isotope Analysis System for SOHO, Solar Phys., 162, 441-481, 1995.

Hughes, W. J. and Engebretson, M. J.: Maccs: Magnetometer Array for Cusp and Cleft Studies, Satellite-Ground Based Coordination Sourcebook, ESA Publications, edited by: Lockwood, M., Wild, M. N., and Opgenoorth, H. J., 1997.

Iyemori, T. and Araki, T.: Single vortex current system in the polar region generated by an interplanetary shock wave, Geophys. Res. Lett., 9(5), 535-538, 1982.

Janhunen, P.: GUMICS-3 A Global Ionosphere-Magnetosphere Coupling Simulation with High Ionospheric Resolution, in: Environment Modeling for Space-Based Applications, edited by: Guyenne, T.-D. and Hilgers, A., vol. 392 of ESA Special Publication, pp. 233-239, 1996.

Kamide, Y. R. and Brekke, A.: Altitude of the Eastward and Westward Auroral Electrojets, J. Geophys. Res., 82(19), 2851-2853, 1977.

Kivelson, M. and Southwood, D.: Ionospheric Traveling Vortex Generation by Solar Wind Buffeting of the Magnetosphere, J. Geophys. Res., 96(A2), 1661-1667, 1991.

Klein, L. W. and Burlaga, L. F.: Interplanetary Magnetic Clouds At 1 AU, J. Geophys. Res., 87(A2), 613-624, 1982.

Kokubun, S., Yamamoto, T., Acuña, M. H. A., Hayashi, K., Shiokawa, K., and Kawano, H.: The GEOTAIL magnetic field experiment, J. Geomagnetism Geoelectricity, 46, 7-21, 1994.

Kullen, A. and Janhunen, P.: Relation of polar auroral arcs to magnetotail twisting and IMF rotation: a systematic MHD simulation study, Ann. Geophys., 22, 951-970, doi:10.5194/angeo-22-9512004, 2004.

Lepping, R. P., Acũna, M. H., Burlaga, L. F., Farrell, W. M., Slavin, J. A., Schatten, K. H., Mariani, F., Ness, N. F., Neubauer, F. M., Whang, Y. C., Byrnes, J. B., Kennon, R. S., Panetta, P. V., Scheifele, J., and Worley, E. M.: The Wind Magnetic Field Investigation, Space Sci. Rev., 71, 207-229, doi:10.1007/ BF00751330, 1995.

Lin, R. P., Anderson, K. A., Ashford, S., Carlson, C., Curtis, D., Ergun, R., Larson, D., McFadden, J., McCarthy, M., Parks, G. K., Rème, H., Bosqued, J. M., Coutelier, J., Cotin, F., D’Uston, C., Wenzel, K.-P., Sanderson, T. R., Henrion, J., Ronnet, J. C., and Paschmann, G.: A Three-Dimensional Plasma and Energetic Particle Investigation for the Wind Spacecraft, Space Sci. Rev., 71, 125-153, 1995.

Mann, I. R., Milling, D. K., Rae, I. J., Ozeke, L. G., Kale, A., Kale, Z. C., Murphy, K. R., Parent, A., Usanova, M., Pahud, D. M., Lee, E.-A., Amalraj, V., Wallis, D. D., Angelopoulos, V., Glassmeier, K.-H., Russell, C. T., Auster, H.-U., and Singer, H. J.: The Upgraded CARISMA Magnetometer Array in the THEMIS Era, Space Sci. Rev., 141, 413-451, 2008.

McComas, D. J., Bame, S. J., Barker, P., Feldman, W. C., Phillips, J. L., Riley, P., and Griffee, J. W.: Solar Wind Electron Proton Alpha Monitor (SWEPAM) for the Advanced Composition Explorer, Space Sci. Rev., 86, 563-612, doi:10.1023/A: 1005040232597, 1998.

Mukai, T., Machida, S., Saito, Y., Hirahara, M., Terasawa, T., Kaya, N., Obara, T., Ejiri, M., and Nishida, A.: The low energy particle (LEP) experiment onboard the GEOTAIL satellite : GEOTAIL Instruments and Initial Results. II, J. Geomagnetism Geoelectricity, 46, 669-692, 1994.

Paschmann, G. and Daly, P. W.: Analysis Methods for MultiSpacecraft Data, The International Space Science Institute (ISSI), 2000.

Pulkkinen, T. I., Partamies, N., Huttunen, K. E. J., Reeves, G. D., and Koskinen, H. E. J.: Differences in geomagnetic storms driven by magnetic clouds and ICME sheath regions, Geophys. Res. Lett., 34, L02105, doi:10.1029/2006GL027775, 2007.

Rème, H., Aoustin, C., Bosqued, J. M., Dandouras, I., Lavraud, B., Sauvaud, J. A., Barthe, A., Bouyssou, J., Camus, Th., CoeurJoly, O., Cros, A., Cuvilo, J., Ducay, F., Garbarowitz, Y., Medale, J. L., Penou, E., Perrier, H., Romefort, D., Rouzaud, J., Vallat, C., Alcaydé, D., Jacquey, C., Mazelle, C., d'Uston, C., Möbius, E., Kistler, L. M., Crocker, K., Granoff, M., Mouikis, C., Popecki, M., Vosbury, M., Klecker, B., Hovestadt, D., Kucharek, H., Kuenneth, E., Paschmann, G., Scholer, M., Sckopke, N., Seidenschwang, E., Carlson, C. W., Curtis, D. W., Ingraham, C., Lin, R. P., McFadden, J. P., Parks, G. K., Phan, T., Formisano, V., Amata, E., Bavassano-Cattaneo, M. B., Baldetti, P., Bruno, R., Chionchio, G., Di Lellis, A., Marcucci, M. F., Pallocchia, G., Korth, A., Daly, P. W., Graeve, B., Rosenbauer, H., Vasyliunas, V., McCarthy, M., Wilber, M., Eliasson, L., Lundin, R., Olsen, S., Shelley, E. G., Fuselier, S., Ghielmetti, A. G., Lennartsson, W., Escoubet, C. P., Balsiger, H., Friedel, R., Cao, J.-B., Kovrazhkin, R. A., Papamastorakis, I., Pellat, R., Scudder, J., and Sonnerup, B.: First multispacecraft ion measurements in and near the Earth's magnetosphere with the identical Cluster ion spectrometry (CIS) experiment, Ann. Geophys., 19, 1303-1354, doi:10.5194/angeo19-1303-2001, 2001. 
Smith, C. W., L'Heureux, J., Ness, N. F., Acuña, M. H. A., Burlaga, L. F., and Scheifele, J.: The ACE Magnetic Fields Experiment, Space Sci. Rev., 86, 613-632, doi:10.1023/A:1005092216668, 1998.

Stauning, P. and Troshichev, O. A.: Polar cap convection and PC index during sudden changes in solar wind dynamic pressure, J. Geophys. Res., 113, A08227, doi:10.1029/2007JA012783, 2008.

Sugiura, M.: Hourly values of equatorial Dst for the IGY, Ann. Int. Geophys. Yr., 35, 9-45, 1964.
Troshichev, O. A., Andrezen, V. G., Vennerstrom, S., and FriisChristensen, E.: Magnetic activity in the polar cap - A new index, Planet. Space Sci., 36, 1095-1102, 1988.

Vanhamäki, H., Amm, O., and Viljanen, A.: 1-Dimensional upward continuation of the ground magnetic field disturbance using spherical elementary current systems, Earth Planets Space, 55, 613-625, 2003.

Yumoto, K. and the CPMN Group: Characteristics of Pi 2 magnetic pulsations observed at the CPMN stations: A review of the STEP results, Earth Planets Space, 53, 981-992, 2001. 